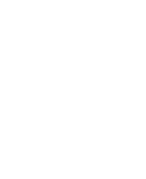
ANNUAL Further
REVIEWS

Click here for quick links to Annual Reviews content online, including:

- Other articles in this volume

- Top cited articles

- Top downloaded articles

- Our comprehensive search

Annu. Rev. Ecol. Evol. Syst. 2011. 42:91-110

First published online as a Review in Advance on August 15, 2011

The Annual Review of Ecology, Evolution, and Systematics is online at ecolsys.annualreviews.org

This article's doi:

10.1146/annurev-ecolsys-102710-145017

Copyright (c) 2011 by Annual Reviews.

All rights reserved

$1543-592 X / 11 / 1201-0091 \$ 20.00$

\section{An Evolutionary Perspective on Self-Organized Division of Labor in Social Insects}

\section{Ana Duarte, ${ }^{1}$ Franz J. Weissing, ${ }^{1}$ Ido Pen, ${ }^{1}$ and Laurent Keller ${ }^{2}$}

\author{
${ }^{1}$ Department of Theoretical Biology, Center for Ecological and Evolutionary Studies, \\ University of Groningen, Groningen, 9747 AG The Netherlands; \\ email: a.l.f.duarte@rug.nl, f.j.weissing@rug.nl, i.r.pen@rug.nl \\ ${ }^{2}$ Department of Ecology and Evolution, University of Lausanne, Lausanne, CH-1015 \\ Switzerland; email: Laurent.Keller@unil.ch
}

\section{Keywords}

behavioral specialization, multilevel selection, emergent properties, caste

\begin{abstract}
Division of labor is a complex phenomenon observed throughout nature. Theoretical studies have focused either on its emergence through selforganization mechanisms or on its adaptive consequences. We suggest that the interaction of self-organization, which undoubtedly characterizes division of labor in social insects, and evolution should be further explored. We review the factors empirically shown to influence task choice. In light of these factors, we review the most important self-organization and evolutionary models for division of labor and outline their advantages and limitations. We describe ways to unify evolution and self-organization in the theoretical study of division of labor and recent results in this area. Finally, we discuss some benchmarks and primary challenges of this approach.
\end{abstract}




\section{INTRODUCTION}

Division of labor is characterized by the performance of different tasks or roles by different groups of individuals. Early economists such as Adam Smith argued that the benefits of division of labor for industrialized societies included increased efficiency in task performance owing to specialization (exclusive dedication of individuals to specific tasks) and reduced costs of switching between tasks, thereby leading to overall higher productivity (Smith 1776).

In nature, division of labor, in the broad sense of the expression, is widespread. The main evolutionary transitions, such as those from prokaryotes to eukaryotes and from unicellular to multicellular organisms, were accompanied by division of labor (Szathmáry \& Maynard Smith 1995). Within social groups division of labor is also common. In species with biparental care, males and females frequently have different roles in raising the offspring. In hornbills, for example, breeding females seal themselves in the nest, and males must feed them during this time (Kemp \& Woodcock 1995). Group hunting (e.g., Gazda et al. 2005), sentinel behavior in group foragers such as meerkats (Manser 1999), and specialization in either predator defense or provisioning in noisy miners (Arnold et al. 2005) are other examples of division of labor.

The most striking example of division of labor in nonhumans occurs in insect societies. We focus on eusocial insects throughout this review owing to the abundant work in this area, although the general principles discussed are equally relevant for other social systems. In eusocial insects, such as honey bees, social wasps, ants, and termites, a few individuals (the queen caste) monopolize reproduction, whereas the rest of the colony (the worker caste) performs tasks such as brood care, nest maintenance, and foraging (Wilson 1971). The success of a colony is determined by its workers' ability to distribute themselves efficiently among tasks and to respond, as a group, to environmental conditions (Gordon 1996, Oster \& Wilson 1978).

Division of labor also occurs among sterile workers. Similar to somatic tissues in a multicellular organism, workers can specialize in different tasks (Oster \& Wilson 1978). Importantly, the concept of specialization is a statistical one, reflecting an individual's tendency to perform particular tasks more often than others. The strength of this tendency may vary greatly, ranging from temporary behavioral differentiation to fixed morphological differentiation in insect species that form large societies (Robinson 1992).

Proximate analyses of division of labor generally are based on the concept of self-organization; according to this view, division of labor is an emergent property of the interaction of individuals obeying simple behavioral rules (Bonabeau et al. 1997, Page \& Mitchell 1998). This concept has been supported by behavioral experiments showing that normally solitary seed-harvester ant queens and halictine bees exhibit task specialization when forced to associate, i.e., paired individuals dedicated most of their time to different tasks (Fewell \& Page 1999; Jeanson et al. 2005, 2008). However, it is unlikely that such behavior would be evolutionarily stable if it occurred over many generations under natural conditions, as the performance of costly tasks that reduce individual fitness would be counterselected. Hence, understanding the proximate mechanisms behind division of labor is not enough; we must also take into account the evolutionary trajectories (ultimate explanation) that selected these mechanisms.

The interplay of self-organization and evolution and the need for integration of the two perspectives have been recognized both inside and outside the scope of social insects (Bonabeau et al. 1997, Corning 1995, Halley \& Winkler 2008, Page \& Mitchell 1998, Richardson 2001, Seeley 2002). However, few models have attempted to unify these two approaches. In this review we outline suggestions to this end. We start by reviewing the empirical evidence on patterns of division of labor in social insects. We then classify existing self-organization models by the factors that create consistent individual differences in task choice in these models and summarize the most 
important evolutionary models for division of labor. We specify the reasons to integrate these selforganization models with an evolutionary perspective and identify the different benchmarks of evolved mechanisms underlying division of labor. Finally, we summarize the first insights obtained from such an integrated perspective and discuss future avenues of research.

\section{EMPIRICAL BACKGROUND}

In this section we first review the primary factors known to contribute to interindividual differences in task choice. Then we discuss how colonies as a whole adjust to environmental changes as well as changes in the size and structure of the workforce.

\section{Worker Size and Morphology}

Worker size and morphology correlate with worker behavior in almost all species in which this has been studied (e.g., Detrain \& Pasteels 1991, Robinson et al. 2009a, Wilson 1980). For example, in ants of the genus Pheidole with discrete morphological castes, the large "majors" are specialized in carrying large prey items to the nest but rarely engage in other tasks such as brood care, which is typically performed by the small "minors" (Mertl \& Traniello 2009, Wilson 1985). It is not too surprising that morphologically differentiated castes behave differently (although empirical evidence indicates that behavior of such castes is flexible to some extent, e.g., Detrain \& Pasteels 1991). However, division of labor also occurs in the absence of such morphological differentiation, and these are the most interesting cases for this review.

\section{Genetic Variation}

In species with multiply mated queens such as honey bees and leaf-cutter ants, different patrilines differ in their tendencies to perform certain tasks (e.g., Julian \& Fewell 2004, Robinson \& Page 1989, Waddington et al. 2010; but see Fournier et al. 2008). Similar results were found for different matrilines in ant species in which colonies contain several queens (Blatrix et al. 2000, Snyder 1992) and in an experiment in which broods of several queens were mixed within a single ant colony (Stuart \& Page 1991). However, almost no information is available about whether the presence of several patrilines or matrilines within a colony is associated with a higher degree of specialization. Although increased within-colony genetic diversity may increase resistance to parasites (e.g., Baer \& Schmid-Hempel 1999, Tarpy 2003, Wilson-Rich et al. 2009), there is little evidence that it leads to increased colony efficiency (Fournier et al. 2008, Rosset et al. 2005).

\section{Developmental and Nutritional Factors}

In many social insects, specialization strongly associated with the age of workers is observed, a phenomenon known as age polyethism (Oster \& Wilson 1978, Wilson 1971). For example, young honey bee workers specialize in in-hive tasks and then switch to foraging later in life. The transition from in-hive worker to forager is associated with physiological alterations, such as changes in juvenile hormone and vitellogenin titers, that correlate with age (Robinson 1987). Worker-worker interactions also mediate changes in hormonal titers through transference of foraging-inhibiting substances (Huang \& Robinson 1996, Leoncini et al. 2004). Nutritional state also plays a role in behavioral differentiation and interacts with developmental hormones and the insulin-signaling pathway (Amdam et al. 2003, Ament et al. 2008, Page \& Amdam 2007, Schulz et al. 1998). 
External factors such as temperature, light, and humidity during development may also affect task preferences. In the ant Camponotus rufipes, temperature during larval development influences the response of individuals to thermal stimuli at the adult stage (Weidenmuller et al. 2009), which causes interindividual differences in tendency to move brood at certain temperatures. Other external factors (e.g., light and humidity) remain to be investigated as sources of variation in task preference.

\section{Individual Experience}

Individual experience influences task preference in the thelytokous (females are produced from unfertilized eggs) ant Cerapachys biroi (Ravary et al. 2007). In individuals of the same age cohort, foraging tendency was positively correlated with exposure to successful foraging experiences in the past. Behavioral differentiation of individuals thus resulted from experience alone. It would be interesting to investigate whether a similar pattern occurs in nonthelytokous species.

\section{Interactions with the Environment}

Social insect colonies have a strong ability to overcome environmental perturbations by changing the number of workers engaged in specific tasks. In harvester ants, the number of foragers shifts as a response to predation risk (MacKay 1982, Munger 1984) or changes in resource availability (Gordon 1991, Schafer et al. 2006). Colonies of the ant Pheidole morrisi respond to seasonal fluctuations in food availability both by individually increasing fat storage and by increasing the number of workers in the replete caste (Yang 2006).

In many species whole colonies readily migrate when the current nest location is damaged or a better location is found. This complex process is similar in honey bees and several ant species (reviewed in Visscher 2007): First, a few individuals scout for new nest sites; second, one location is chosen through quorum sensing (Pratt 2005, Seeley \& Morse 1978); and third, the whole colony moves to the new location.

Social interactions are crucial to efficiently react to environmental change. Depending on species, active workers can use chemical signals or direct physical contact to recruit inactive workers. Contact with successfully returning foragers influences the decision of ants in the nest to forage or not in several species (Gordon \& Mehdiabadi 1999; Greene \& Gordon 2003, 2007; Robinson et al. 2009b). Foraging honey bees, upon returning to the nest, must search for foodstorer bees that unload and store the nectar collected. The time spent searching for food-storer bees has been shown to be used by foragers to regulate foraging rates; short search times elicit the recruitment of more foragers (Seeley \& Tovey 1994). The modulation of division of labor by worker-worker interactions thus appears to allow for efficient use of the information acquired by all workers in the colony.

\section{Colony Size and Life Cycle}

Eusocial insect colonies typically go through three different phases in their life cycle: a founding stage in which new nests are founded by recently mated queens (and kings, in the case of termites), an ergonomic growth stage in which the colony produces workers only and increases in size, and a reproductive stage in which the colony produces new sexuals (males and gynes) (Oster \& Wilson 1978). The transition from ergonomic growth to reproduction is generally marked by the attainment of a certain colony size, which varies from species to species.

In species with morphological castes, often in the initial founding stage the queen produces only small workers ("minims"), and as the colony grows, the average worker size increases (Hölldobler 
\& Wilson 1990). Throughout the lifetime of a colony, the need for some tasks, such as nest building and foraging, should drastically change, although the need for other tasks such as maintaining temperature and humidity conditions should remain similar. In Lasius niger ants, different proportions of workers are engaged in particular tasks over the growth of the colony (Mailleux et al. 2003). However, to our knowledge no empirical study has measured the extent of changes in task need with colony growth.

Colony size has been argued to influence the complexity of social behavior and division of labor; smaller colonies should have more generalist workers (Anderson et al. 2001, Bourke 1999, Karsai \& Wenzel 1998). For example, in the ant Rhytidoponera metallica, large colonies show pronounced age-based division of labor whereas small colonies do not (Thomas \& Elgar 2003). In the ant Temnothorax albipennis, large colonies have larger proportions of brood-carrying specialists than small colonies (Dornhaus et al. 2008). However, there are no differences between large and small colonies in a standardized measure of division of labor (Dornhaus et al. 2009).

\section{MODELS FOR SELF-ORGANIZED DIVISION OF LABOR}

Self-organization models attempt to explain the division of labor at the colony level by using simple individual behavioral rules that assume neither complex cognitive abilities nor centralized control. We classify these models into three groups on the basis of the factors underlying the emergence of individual differences in task choice, namely, signal-response dynamics, spatial differentiation of tasks, and social interactions.

\section{Signal-Response Dynamics}

These models assume that individuals differ in their responses to environmental signals of the colony's need for specific tasks. Possible signals include pheromones emitted by larvae to elicit care from workers, nest temperature, or the amount of stored food. The models further assume that these signals are dynamic: Signals increase in intensity in the absence of sufficient task performance and decrease with a certain level of task performance (Figure 1 $\mathbf{a}$ ).

The fixed threshold models assume that each individual has thresholds for particular tasks that are constant over time. Thresholds determine the intensity of signal needed for the individual to respond (Bonabeau et al. 1996, 1998; Gove et al. 2009; Graham et al. 2006; Jeanson et al. 2007; Page \& Mitchell 1998). If signal intensity lies above an individual's threshold, the individual will perform the task. If signal intensity lies below the individual's threshold, the individual will not perform the task. The feedback between task performance and signal intensity allows for consistent interindividual differences to be expressed: Individuals with lower thresholds for a task decrease the signal for that task, thereby decreasing the probability that individuals with higher thresholds will ever perform it. However, individuals may eventually perform tasks for which they have high thresholds in the absence of workers with lower thresholds. Hence, both task specialization and flexibility can be emergent outcomes of the model's dynamics.

The reinforced threshold model uses the same concept, with the difference that individual thresholds can change owing to experience (Gautrais et al. 2002, Plowright \& Plowright 1988, Theraulaz et al. 1998). Performance of a task decreases the corresponding threshold, whereas not performing a task results in an increase of the threshold.

The empirical support for a response threshold-like mechanism comes from studies of thermoregulation in bumble bees and ants in which different individuals consistently started to perform thermoregulatory behaviors at different temperatures (O'Donnell \& Foster 2001, Weidenmuller 2004). Thresholds likely have a genetic or developmental basis, because different morphological 


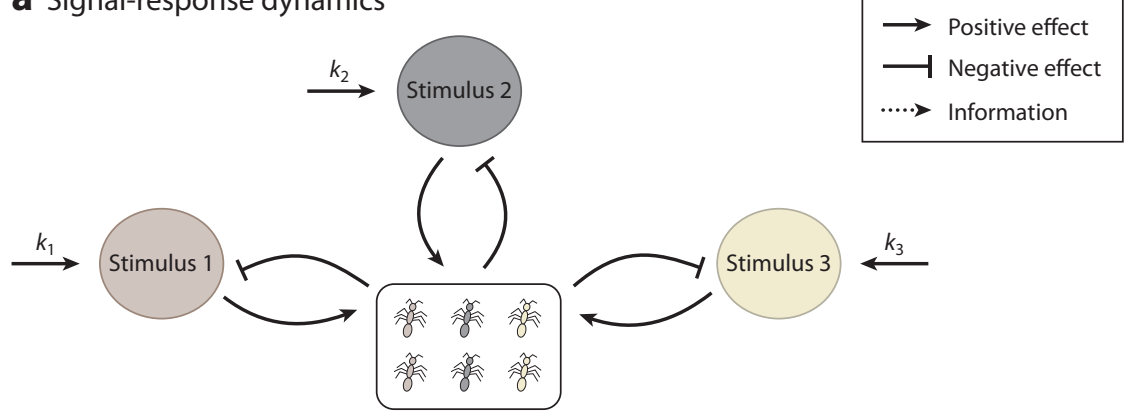

\section{b Spatial differentiation}

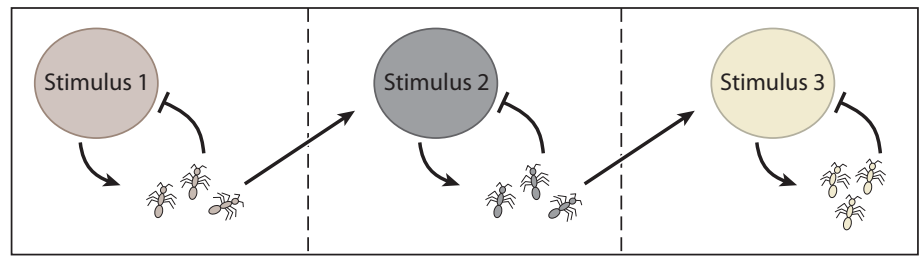

\section{Social interactions}

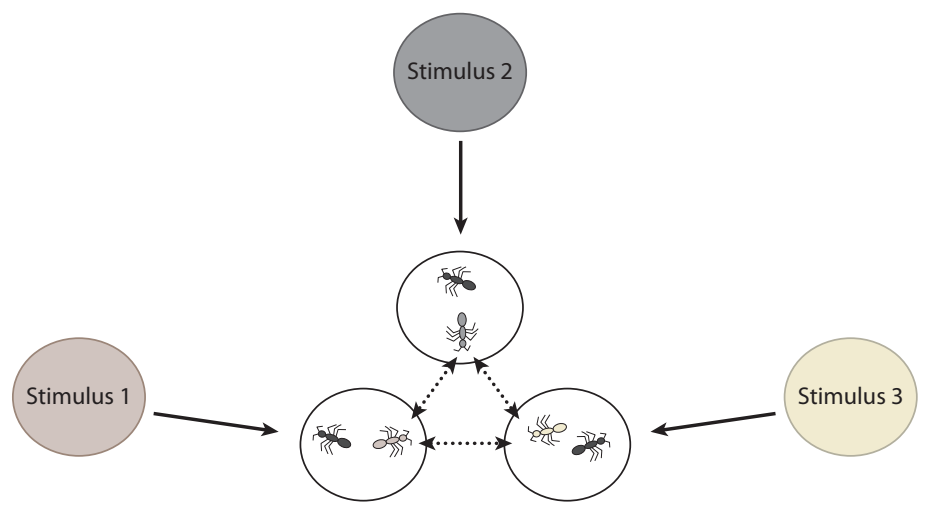

Figure 1

Schematic representation of the main factors contributing to interindividual variation in self-organization models of division of labor. (a) In signal-response models, the number of active workers increases with the amount of stimulus (increasing by $k$ over time), and active workers reduce the stimulus level, thus decreasing the need for more workers to become active. Intrinsic differences in workers, such as response thresholds, are indicated by different colors. (b) Spatial differentiation of tasks (and of the associated stimuli) leads to different individual behavior in the foraging-for-work model (drawn from information in Tofts 1993). The local stimulus determines the number of active workers for each task (curved arrows). Task-related items are processed and passed from one task group to another (straight arrows), which promotes the increase of stimulus at the different locations. Workers active for different tasks are indicated in different colors. $(c)$ In social interaction models, the decision of workers to perform different tasks is affected not only by the associated stimuli (solid arrows) but also by information received from other workers (dashed arrows). Workers active for different tasks are indicated in the colors of the corresponding tasks. Idle workers are depicted in black. 
castes as well as genetic lineages generally differ in their tendency to respond to task-associated stimuli (Detrain \& Pasteels 1991, Page et al. 1998, Pankiw et al. 1998). Ravary and coworkers (2007) provide empirical support to the threshold reinforcement model.

Owing to our lack of knowledge about the actual dynamics of task stimuli in real colonies, the implementation of task stimuli in signal-response models is simplistic. Many of these models assume a linear increase in task stimulus in the absence of work and a linear decrease with the amount of work performed on the task, but stimulus increase or decrease will almost certainly be nonlinear in a real-world setting. The nature of tasks may also differ: Some tasks require the maintenance of the stimulus within certain bounds (homeostatic tasks, e.g., thermoregulation), whereas for other tasks it is adequate to keep the stimulus at the lowest level (maximizing tasks, e.g., foraging). The type of tasks present may influence the extent to which workers should specialize or generalize (Johnson 2003).

\section{Spatial Differentiation}

The foraging-for-work model (Franks \& Tofts 1994, Tofts 1993) suggests that the spatial distribution of tasks could lead to interindividual differences in behavior. In this model, tasks are spatially distributed, similar to a production line. Individuals at each location process and pass along items coming from an external source until the items reach the final task in the line (Figure 1 b). This idea takes inspiration from real colonies in which individuals forage, cut up food items, and transport them back to the colony, where finally the food items are fed to the brood.

The algorithm of task choice in the standard versions of the foraging-for-work model (Franks \& Tofts 1994, Tofts 1993) requires that individuals actively look for another task if they fail to pass an item to the subsequent task or if they fail to receive items from the preceding task. At the equilibrium state, workers stay in the same task for a prolonged time. By including in the model a certain rate at which individuals are born and introduced into the workforce at the last task in the production line (analogous to brood care) and a maximal age after which workers die, age polyethism emerges. This is due both to the fact that younger workers displace older workers in their task and to the assumption that tasks are spatially distributed in a sequence.

The foraging-for-work model aims to show that task choice need not be state or age dependent for age polyethism to occur. The assumption that all workers have equal tendencies to perform every task has encountered some criticism (Robinson et al. 1994, Traniello \& Rosengaus 1997) because much evidence has been found for both genetic and physiological correlates with task choice (see previous section). Recent evidence also indicates that young workers are able to do fewer tasks than older workers (Seid \& Traniello 2006).

\section{Social Interactions}

Interaction models explore the role of social interactions as the driving mechanism behind division of labor. Interactions among workers lead to shifts in their behavioral state and task choice. The models differ in the information exchanged during interactions.

Gordon et al. (1992) assume that workers, via social interactions, receive information about tasks performed by others. By comparing the perceived task distribution with an intrinsic desired task distribution, individuals switch tasks in the case of a mismatch between the two distributions. As a result, an equilibrium is reached in which workers distribute themselves according to the intrinsic task distribution.

Pacala et al. (1996) develop a model in which the profitability of tasks changes with time. By comparing the perceived profitability of others with the profitability of their own task, workers 
decide whether to switch to a more profitable task. If profitability is proportional to colony efficiency, this process leads to a colony that is able to track environmental variation, thereby maintaining an efficient distribution of workers over tasks.

Other models address more specifically the division of labor between nest workers and foragers as well as its modulation by inhibitory interactions between the two types of workers (Beshers et al. 2001, Naug \& Gadagkar 1999). These models find that a simple social activation-inhibition mechanism can explain the pattern of age polyethism often found in eusocial insects.

\section{Limitations of Current Self-Organization Models}

By demonstrating that emergent specialization is possible through simple mechanisms, selforganization models serve as a proof of principle that high cognitive processes are not required to achieve complex group behavior. However, these models typically do not consider the evolutionary trajectories that may lead to division of labor. This also holds for those self-organization models that implicitly incorporate adaptive reasoning. For example, in social interaction models, task choice algorithms are designed to favor tasks for which there is a higher need. However, the question of why and how individuals are endowed with an intrinsic knowledge of colony needs is not addressed. Without a clear idea of how natural selection shaped the task choice mechanism underlying a self-organization model, our understanding of self-organized division of labor remains incomplete. Bonabeau et al. (1997, p. 191) stated: “...natural selection, operating on parameters that modulate individual and colony-level properties, has certainly picked the forms of self-organization that we see in social insects because they are adaptive or cooperative." However, disappointingly few attempts have been made to develop realistic scenarios for how the mechanisms underlying self-organized division of labor evolve over the course of generations.

\section{EVOLUTIONARY MODELS OF DIVISION OF LABOR}

\section{Optimal Caste Ratios}

The first generation of evolutionary models for division of labor applied optimality principles to understand the distribution of castes and tasks in a colony. In their classic book, Oster \& Wilson (1978) derived optimal caste ratios in a situation in which morphological differences influenced the efficiency with which individuals could perform various tasks. They offer two primary theoretical explanations for the observation that in social insects fewer castes than tasks exist: First, production of a broad variety of physical castes is costly at the colony level; second, the behavioral flexibility of castes allows the colony to react more rapidly to environmental changes.

Oster \& Wilson's view of caste ratios as an adaptive trait has been challenged by empirical studies that consistently fail to find correlations between colony productivity and caste ratios (for a review see Schmid-Hempel 1992). The behavioral flexibility of workers is considered to be the main factor responsible for adjustment of the worker force to changing environments, including different ratios of morphological or age castes (Gordon 1996). Nevertheless, the question still remains of whether the existence of different morphological castes actually increases fitness and, if so, why morphological castes are not found more often.

\section{Adaptive Specialization}

Any form of specialization may be beneficial for two reasons (Smith 1776): First, it may allow for higher individual efficiency (e.g., owing to training or through gains in task-specific information); second, specialization may reduce the costs of switching tasks (e.g., time lost traveling between 
task locations or energy costs owing to shifts in behavioral state). Several evolutionary models of division of labor have investigated the conditions under which a task-specialist strategy is favored over a task-generalist strategy.

Wakano and coworkers (1998) developed an age polyethism model in which workers were grouped into different age classes. Each age class allocated labor in predetermined proportions for inside (e.g., brood care) and outside (foraging) tasks. In a situation in which outside tasks incurred higher mortality risk and thus worker life expectancy decreased with age, a strict age polyethism (with young workers specializing in inside tasks and older workers specializing in outside tasks) was adaptive. Furthermore, environmental fluctuations affected the type of age polyethism that was optimal: When large fluctuations alter the efficiency of both inside and outside workers, a smooth transition from performing inside to outside tasks is optimal. When fluctuations affected only foraging, a sharp transition was favored.

Several other models have been proposed to explain division of labor in systems other than social insects. Wahl (2002) formulated simple game-theory models to study division of labor in coviruses, in which different types of virions coinfect a host cell and perform complementary tasks in the replication process (Nee 1987). In these models, phenotypes were fixed: Individuals could either perform only one of the tasks (specialists) or have the ability to perform all tasks (generalists). If all tasks were performed, individuals shared the resulting benefits. Wahl made two types of assumptions concerning the costs associated with task performance. She found that when costs were paid mainly for the ability to perform a task (fixed costs), rather than for the performance itself (marginal costs), the outcome was a polymorphic population of specialists, i.e., individuals who could perform complementary tasks. When costs were paid for task performance only, a combination of generalists and specialists for one task was optimal.

Although Wahl's models were inspired by a different context, some parallels to social insects can be drawn. In social insects we could consider as fixed the costs involved in developing particular morphological or physiological structures, or the costs involved in switching tasks. These are costs that individuals pay for the ability to perform a task. Marginal costs would be, for example, the energy expenditure involved in task performance. From Wahl's results, one could thus predict that separate groups of specialists should frequently evolve when fixed costs are involved, such as the costs of developing features such as strong mandibles or storage organs.

Another model for studying the adaptive value of specialization takes inspiration from enzymesubstrate reactions. Tannenbaum (2007) compared the productivity of systems in which binding and processing a resource to release a final product could be performed through an undifferentiated pathway (generalist strategy) or a differentiated pathway (specialist strategy). For a low density of agents, undifferentiated pathways were favored owing to the costs of transferring products between specialists. In a resource-limited regime, a differentiated pathway was more productive than an undifferentiated pathway when the specialist enzymes were more efficient at binding to the incoming resource and process intermediate. When resource availability was high, the differentiated pathway became more productive when it could process faster the molecules to which agents were bound. These results are quite intuitive because at low densities, transferring the products of tasks among specialists becomes less efficient, and hence division of labor is selected against. In social insects this should translate into less marked division of labor in smaller colonies, as indeed seems to be the case (Thomas \& Elgar 2003).

\section{Limitations of Current Evolutionary Models}

The main limitation of current evolutionary models is that they tend to ignore the mechanisms through which specialization may arise. Using a fixed set of strategies, these models use fitness and 
productivity measures to test when specialization is better than a generalist strategy. Much like selforganization models implicitly assume evolutionary adaptation, evolutionary models assume that some mechanism will evolve that allows for adaptive behavior. The ability to evolve specialization, however, may be strongly dependent on the mechanism through which it arises. Furthermore, the analysis of only a fixed set of strategies is limiting. A better approach would be to allow the set of strategies to evolve itself, thereby alleviating the constraints on adaptive evolution.

Specialization is the crucial defining property of division of labor. Evolutionary models are usually based on the (plausible) assumption that worker specialization enhances the productivity of a colony because of synergistic effects. However, surprisingly little information is available on how specialization increases productivity. Moreover, the information available does not suggest a close correlation between individual efficiency in task performance and specialization (Dornhaus 2008, Muscedere et al. 2009).

\section{UNIFICATION OF SELF-ORGANIZATION AND EVOLUTIONARY MODELS}

In our view, self-organization and evolutionary models are not contradictory in any way, and in fact they can (and should) be integrated into a common framework. One way to do this is illustrated in Figure 2. The figure shows that it is useful to distinguish between two timescales: a short-term

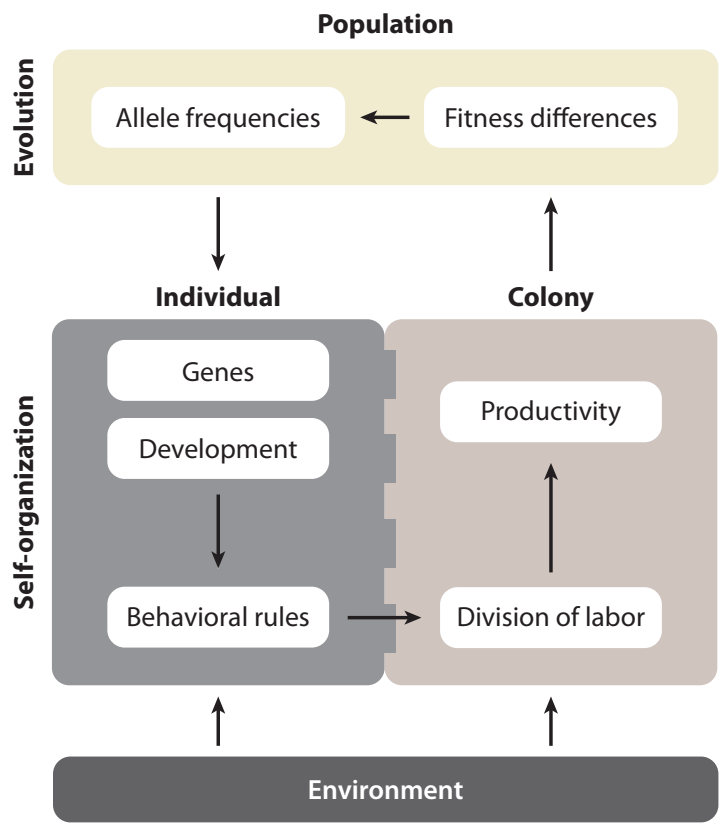

Figure 2

Integration of self-organization and evolution in a coherent framework. On a short-term timescale, self-organization takes place. The factors influencing task choice at the individual level (i.e., genetics, development, and environment—abiotic and biotic, including social interactions) interact to produce the individual phenotype, the rules through which individuals self-organize to divide labor. The colony's behavior, and its interaction with the environment, has an effect on productivity and hence on fitness. On the long-term timescale, natural selection acts on the existing variation in fitness at the population level, thereby changing allelic frequencies of the genes underlying the rules for self-organized behavior. 
timescale at which interactions take place (corresponding to the life of a colony) and a long-term timescale at which evolutionary changes occur (Figure 2). On the short-term timescale, selforganized division of labor emerges as a result of the behavior of individuals, who obey inherited behavioral rules. Division of labor then has a direct relationship with productivity of a colony and fitness (number of reproductives). On the long-term timescale, fitness differences between colonies lead to a change in allele frequencies and hence in the genetic makeup of individuals, eventually changing the rules underlying division of labor.

A first approach is to consider various task choice algorithms, implement a measure of colony fitness, and allow for selection among the algorithms. A good example of this approach is the model of Waibel et al. (2006). They implemented a task allocation model in which colony performance increased with the number of workers engaged in each task. Different task choice algorithms were studied, and their underlying genes were allowed to evolve under different conditions of intracolonial genetic relatedness and environmental perturbations. Under environmental perturbations and high relatedness, a more flexible algorithm that took both individual preferences and the behavior of colony members into account performed best. Unfortunately, the implications of this model for division of labor cannot be evaluated because individual levels of specialization were not reported.

In a similar study, Tarapore et al. (2009) implemented an evolutionary version of the fixed response threshold model in which thresholds were allowed to evolve in order to study the genetic architecture and mating system in a task allocation scenario. Individuals could perform a foraging task and a regulatory task; fitness benefits from the foraging task accrued only when the regulatory task was within certain bounds. The colonies achieving the highest performance in each generation contributed to the next generation in proportion to their fitness. The results of this study indicate that variation in thresholds is a main determinant of colony productivity. Hence, colony performance was influenced by the number of males queens mated with as well as the number of loci encoding the response thresholds.

A. Duarte, I. Pen, L. Keller \& F.J. Weissing (submitted) also implemented an evolutionary version of the fixed response threshold model in which the evolutionary trajectories of the thresholds were explicitly followed. The model studied the effect of the evolution of thresholds on division of labor, colony fitness, and work distribution. This study demonstrated that specialization could evolve if switching among tasks involved high costs. If this was the case, a previously homogeneous population evolved to a multimodal distribution of thresholds (via evolutionary branching), and differentiation of thresholds allowed for division of labor. However, only colonies with a correct combination of thresholds exhibited pronounced specialization. The branching of thresholds also can be interpreted as the evolution of separate morphological castes in which individuals of a caste behave as specialists if enough individuals of the other caste are present, as also observed in empirical data (Wilson 1985).

The above models are limited because they take an existing self-organization model (similar to the response threshold model) as their point of departure. This imposes limitations upon the mechanisms that can potentially evolve and therefore constrains the path of evolution. For example, in the model by Tarapore et al. (2009), individuals generally evolved low thresholds for the regulatory task, whereas thresholds for the foraging task were highly variable among individuals. Hence, when the stimulus for the regulatory task was low, workers with a high threshold for the foraging task remained idle. Thus, colony efficiency was constrained by the inability to evolve thresholds that minimize idleness for a given task independent of the stimulus level for the other task. Similarly, A. Duarte, I. Pen, L. Keller \& F.J. Weissing (submitted) noticed that the standard implementation of the fixed threshold model always leads to a uniform distribution of workers over tasks, even if a skewed distribution is more adaptive. In fact, the distribution of workers over 


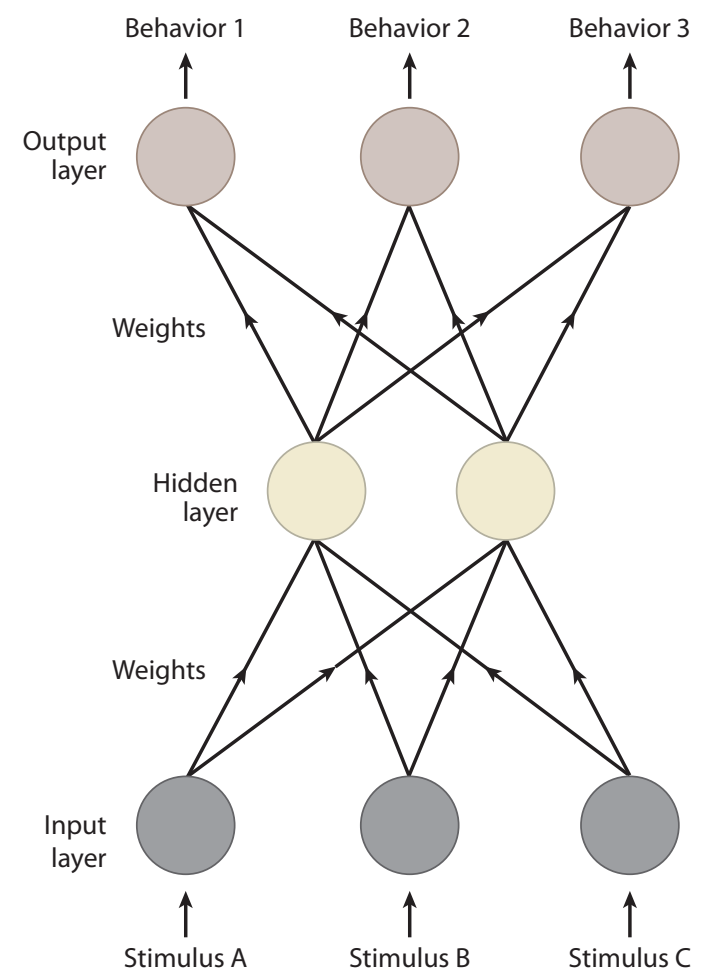

Figure 3

Example of a simple feed-forward neural network. Environmental stimuli are perceived by neurons at the input layer and passed on as signals to the hidden layer, where they are further transformed. Finally, the signal reaches the output neurons, where a decision is made regarding which task to perform. The network has many parameters, such as connection weights (arrows), that can be positive or negative and influence the strength of the signal. These parameters are allowed to evolve.

tasks was largely independent of the location of the thresholds; it instead was determined by the parameters governing the stimulus dynamics.

A possible way to circumvent the limitations of a priori mechanisms is to investigate the evolution of more flexible task choice mechanisms. One interesting option is neural network modeling, which is applied regularly in evolutionary robotics and has offered interesting insights into the evolution of behavioral strategies such as communication and cooperation (Floreano \& Keller 2010; Floreano et al. 2007; Mitri et al. 2009, 2011; Perez-Uribe et al. 2003). Neural networks can be used to simulate the processing of stimuli by an individual through receptor cells connected to output cells controlling behavioral responses (Figure 3) (Ghirlanda \& Enquist 1998). Even for relatively small neuronal networks, the number of potential stimulus-response patterns (which form the substrate for evolution) is almost limitless. All kinds of behavioral architectures can evolve, whereas in traditional models the behavioral bauplan is determined by the modeler a priori. On the downside, the evolution of neural networks is not always efficient, because fitness optima must be found in a high-dimensional trait space. Moreover, the interpretation of the functioning of an evolved network is not always straightforward.

Two recent studies apply network modeling to the evolution of self-organized division of labor. P. Lichocki, D. Tarapore, L. Keller \& D. Floreano (submitted) considered a network version of Tarapore et al.'s (2009) model for combining a foraging and a regulatory task. They showed that 
the efficiency of task allocation and overall colony productivity were greatly improved when a simple neural network controlled individual decisions. In the evolved networks, the link between the stimulus for one task and the likelihood of performing the other task was partly decoupled. As a result, fewer workers were idle, which led to higher colony efficiency.

In the same vein, in the model of A. Duarte, E. Scholtens \& F.J. Weissing (submitted), a neural network approach allowed for worker distribution over tasks to be more flexible than in the fixed response threshold model. This study also investigated a slightly more complex network that allows past experience to affect individual behavior. Specialization mediated by individual experience evolves easily when switching tasks bears costs to the colony.

\section{Benchmarks of Evolved Mechanisms Underlying Division of Labor}

In this section we highlight some desirable features that a unifying model for the evolution of selforganized division of labor should include. We discuss these features in light of existing models and suggest future avenues of research.

Emergent specialization and adaptive distribution of workers. Because emergent specialization at the individual level is the most obvious property of division of labor, we are interested in those systems in which selection favors the establishment of specialization. Specialization may be selected directly, if it brings an immediate advantage, or indirectly, if the behavioral rules leading to specialization are selected owing to other beneficial consequences.

Specialization is easy to obtain in self-organization models, but it may be unstable when studied from an evolutionary perspective. For example, in the model of A. Duarte, I. Pen, L. Keller \& F.J. Weissing (submitted), selection for maximum colony performance often drives thresholds to zero level. As a result, specialization vanishes, even if it is beneficial for colony productivity.

For the proper functioning of the colony as a whole, an evolved mechanism for division of labor should produce an adaptive distribution of workers over tasks. As shown by A. Duarte, I. Pen, L. Keller \& F.J. Weissing (submitted), the standard version of the response threshold model does not have this property, because it always leads to a uniform distribution of workers over tasks. Hence, a more sophisticated modeling setup is required (such as neural network modeling; A. Duarte, E. Scholtens \& F.J. Weissing, submitted) to enable the evolution of an asymmetric distribution in which some tasks are performed by a larger proportion of workers whereas other tasks require fewer individuals.

Flexibility and developmental robustness. Any realistic model for the evolution of division of labor should allow for flexibility in colony behavior, that is, the ability to cope with environmental challenges necessitating a change in the distribution of workers over tasks. Thus far, only the interaction model of Pacala et al. (1996) addresses such flexibility, but even in this model it is unclear how workers acquire knowledge about how the profitability of tasks changes over time. There is an urgent need for models exploring the implications of temporal variation (e.g., seasonal variation in food abundance).

The ability to cope with environmental challenges necessitates robustness with respect to the internal state of the colony. An adequate timing of task performance and an optimal distribution of workers over tasks should be attained irrespective of factors such as within-colony genetic variability, colony demographic structure, or the developmental dynamics of a colony (see Tarapore et al. 2009). Developmental plasticity that allows the generation of phenotypic diversity in colonies with low genetic diversity has not yet received the attention it deserves. 
Evolutionary attainability and stability. It is now well established that in social evolution seemingly favorable traits (such as a mechanism that generates division of labor) are not necessarily attainable because selection may drive a population away from these solutions (Garden of Eden scenarios; Nowak 1990). Even if a seemingly favorable trait is attained, it is not necessarily evolutionarily stable (Maynard Smith 1982). Examples of inaccessible or evolutionarily unstable traits abound in situations with evolutionary conflicts or counteracting selection at different levels of organization (McNamara \& Weissing 2010). Both factors are relevant in colonies of social insects.

Members of a colony may have conflicting interests regarding caste fate of female brood, relative colony investment in males and females, and worker reproduction (reviewed in Ratnieks et al. 2006). Conflict over sex allocation may lead to a tug-of-war between queens and workers in which each party attempts to bias the colony sex ratio toward its optimum (Pen \& Taylor 2005, Reuter \& Keller 2001, Reuter et al. 2004). For example, workers may bias the sex ratio by killing males (e.g., Sundström et al. 1996) or increase the proportion of diploid individuals raised as reproductive females (e.g., Hammond et al. 2002). Queens, in return, may limit the number of available eggs to prevent workers from eliminating males (e.g., Passera et al. 2001). These mechanisms of sex-ratio manipulation by queens and workers are likely to incur costs because of suboptimal investment in workers and/or waste of resources already invested in the males eliminated (Helms et al. 2005, Reuter et al. 2004). Theory also suggests that female larvae are in conflict with both queens and workers regarding their caste fate, and when in control of their development, a high proportion of female larvae should develop into reproductive females instead of sterile workers (Bourke \& Ratnieks 1999, Ratnieks 2001, Reuter \& Keller 2001, Wenseleers et al. 2003).

Because within-colony conflicts can decrease the number of active workers within a colony and change the ratios of worker castes when different types of workers vary in their likelihood to reproduce (Bourke \& Franks 1995), these conflicts may reduce the evolutionary attainability and stability of division of labor. Thus far, models integrating self-organization and evolution have not dealt with selection at multiple levels; this is a point to address in future work.

\section{Challenges}

We end this review with a brief discussion of three aspects of models for the evolution of division of labor that we find particularly challenging.

Fitness. Empirically, little is known about how division of labor affects colony fitness. The relationship between ergonomic efficiency and fitness was one of the basic open questions pointed out by Oster \& Wilson (1978) in their book Caste and Ecology in the Social Insects. Thirty years later, little progress has been made. Current models use proxies for fitness such as the overall work performed and the distribution of workers over tasks. Although these features are undoubtedly related to colony survival, growth, and productivity, they do not necessarily have a simple and straightforward relationship with colony fitness. In fact, predictions based on such components of fitness (rather than on overall fitness) can be highly misleading (e.g., McNamara \& Weissing 2010). In the context of division of labor, it remains a challenge to develop more realistic approximations of the relationship between fitness and task performance, such as the number of reproductive individuals produced by a colony as well as their reproductive values.

Realism of biological details. In the vast majority of models of division of labor, information on task needs is global. However, owing to the spatial distribution of tasks, all group members 
are unlikely to have the same information. In a more realistic setting, information on task needs should be local; group members should transmit information with some noise and cost.

Importantly, concerning the realism of evolutionary parameters, scientists working on selforganized processes at the colony level or evolutionary processes over many generations frequently have different backgrounds (e.g., mathematics, physics, computer science, and engineering for self-organization models and evolutionary biology for ultimate questions) and hence use different approaches. For example, Tarapore et al. (2009) and A. Duarte, I. Pen, L. Keller \& F.J. Weissing (submitted) use a high mutation rate, strong selection, and few generations to minimize computation time. These authors follow the example of artificial intelligence studies in which the goal is to develop fast solutions for highly complex systems in which several dynamic processes co-occur. From the engineering perspective of such studies, it makes sense to set parameters such as mutation rates or recombination probabilities to values that enhance the efficiency of the evolutionary algorithms. In contrast, evolutionary biologists are facing systems with externally given parameters, which may have less desirable properties. It remains to be seen how strongly evolutionary conclusions are dependent on the parameter settings used in simulations.

Comparison of division of labor in different models. Thus far we have treated division of labor as a concept that overlaps with specialization. However, it is desirable to distinguish between individual specialization, which is a property of the individual, and division of labor, which is a property of the group (Gorelick et al. 2004). To better compare different models, a standardization of the measures used for quantifying specialization and division of labor would be desirable.

Specialization has been quantified as the frequency of task performance (Bonabeau et al. 1996) by estimating the proportion of time individuals do specific tasks. This measure, however, does not take into account the pattern of task choice. An individual switching randomly between two tasks and another switching only once during the observed period can achieve the same value of task frequency: They both spend half their time doing one task and the other half doing the other task. Another measure of specialization that is based on the probability of individuals to switch among tasks (Gautrais et al. 2002) produces a much higher value of specialization for the individual that switched tasks only once than for the individual that switched randomly between tasks.

Individual specialization is only part of the story, because we can talk about division of labor only if individuals are specialized in different tasks. Gorelick \& Bertram (2007) suggested several matrix statistics, taking into consideration information on both tasks and individuals, which are useful measures of the nestedness of task choice (i.e., how restricted certain individuals are to certain tasks and vice versa). These statistics, however, do not account for the distribution of workers over tasks. To cope with the fact that each measure on its own offers only an incomplete picture of a model's behavior, it is perhaps necessary to include different measures, including a description of specialization at the individual level, a nestedness measure of tasks and individuals, and an overall task performance ratio.

\section{CONCLUSION}

Unification of evolution and self-organization will certainly enlighten us on what kind of mechanisms can evolve. However, empirically, little is still known about individual differences in behavior within colonies and the proximate mechanisms underlying the propensity of an individual to engage in a given task. The main obstacle to opening this black box lies in the difficulty of tracking individuals within colonies and manipulating their environment. Fortunately, new studies using radio tagging and fiduciary markers have begun to provide insights on the factors affecting the behavior of individuals within colonies and how, in turn, this influences interactions at the colony 
level. The combination of such behavioral analyses with new genomic tools applicable to a wide range of social insects (Graff et al. 2007, Grozinger et al. 2007, Smith et al. 2010, Wurm et al. 2010) should provide insights into how genetic differences and social interactions interact to jointly affect individual behavior and patterns of division of labor at the colony level.

Self-organization models and evolutionary models are two sides of the same coin. On one hand, we must understand the behavioral rules on which self-organization is based; on the other hand, a full understanding of division of labor must encompass the evolutionary trajectories of such rules. We hope that this review encourages more studies including both perspectives in their research agendas.

\section{DISCLOSURE STATEMENT}

The authors are not aware of any affiliations, memberships, funding, or financial holdings that might be perceived as affecting the objectivity of this review.

\section{ACKNOWLEDGMENTS}

A.D. received a Ph.D. grant from the Center for Ecological and Evolutionary Studies, University of Groningen. L.K. is grateful for funding from the Swiss NSF and an advanced ERC grant. The authors would like to thank an anonymous reviewer for comments that greatly improved the manuscript.

\section{LITERATURE CITED}

Amdam GV, Norberg K, Hagen A, Omholt SW. 2003. Social exploitation of vitellogenin. Proc. Natl. Acad. Sci. USA 100(4):1799-802

Ament SA, Corona M, Pollock HS, Robinson GE. 2008. Insulin signaling is involved in the regulation of worker division of labor in honey bee colonies. Proc. Natl. Acad. Sci. USA 105(11):4226-31

Anderson C, Franks NR, McShea DW. 2001. The complexity and hierarchical structure of tasks in insect societies. Anim. Behav. 62:643-51

Arnold KE, Owens IPF, Goldizen AW. 2005. Division of labour within cooperatively breeding groups. Behaviour 142:1577-90

Baer B, Schmid-Hempel P. 1999. Experimental variation in polyandry affects parasite loads and fitness in a bumble-bee. Nature 397(6715):151-54

Beshers SN, Huang ZY, Oono Y, Robinson GE. 2001. Social inhibition and the regulation of temporal polyethism in honey bees. 7. Theor. Biol. 213(3):461-79

Blatrix R, Durand JL, Jaisson P. 2000. Task allocation depends on matriline in the ponerine ant Gnamptogenys striatula Mayr. 7. Insect Behav. 13(4):553-62

Bonabeau E, Theraulaz G, Deneubourg JL. 1996. Quantitative study of the fixed threshold model for the regulation of division of labour in insect societies. Proc. R. Soc. B 263(1376):1565-69

Bonabeau E, Theraulaz G, Deneubourg JL. 1998. Fixed response thresholds and the regulation of division of labor in insect societies. Bull. Math. Biol. 60(4):753-807

Bonabeau E, Theraulaz G, Deneubourg JL, Aron S, Camazine S. 1997. Self-organization in social insects. Trends Ecol. Evol. 12(5):188-93

Bourke AFG. 1999. Colony size, social complexity and reproductive conflict in social insects. F. Evol. Biol. 12(2):245-57

Bourke AFG, Franks NR. 1995. Social Evolution in Ants. Princeton, NJ: Princeton Univ. Press

Bourke AFG, Ratnieks FLW. 1999. Kin conflict over caste determination in social Hymenoptera. Behav. Ecol. Sociobiol. 46(5):287-97

Corning PA. 1995. Synergy and self-organization in the evolution of complex systems. Syst. Res. 12(2):89-121 
Detrain C, Pasteels JM. 1991. Caste differences in behavioral thresholds as a basis for polyethism during food recruitment in the ant, Pheidole pallidula (Nyl) (Hymenoptera, Myrmicinae). F. Insect Bebav. 4(2):157-76

Dornhaus A. 2008. Specialization does not predict individual efficiency in an ant. PLoS Biol. 6(11):e285

Dornhaus A, Holley JA, Franks NR. 2009. Larger colonies do not have more specialized workers in the ant Temnothorax albipennis. Behav. Ecol. 20(5):922-29

Dornhaus A, Holley JA, Pook VG, Worswick G, Franks NR. 2008. Why do not all workers work? Colony size and workload during emigrations in the ant Temnothorax albipennis. Behav. Ecol. Sociobiol. 63(1):43-51

Fewell JH, Page RE. 1999. The emergence of division of labour in forced associations of normally solitary ant queens. Evol. Ecol. Res. 1(5):537-48

Floreano D, Keller L. 2010. Evolution of adaptive behaviour in robots by means of Darwinian selection. PLoS Biol. 8(1):e1000292

Floreano D, Mitri S, Magnenat S, Keller L. 2007. Evolutionary conditions for the emergence of communication in robots. Curr. Biol. 17(6):514-19

Fournier D, Battaille G, Timmermans I, Aron S. 2008. Genetic diversity, worker size polymorphism and division of labour in the polyandrous ant Cataglyphis cursor. Anim. Behav. 75:151-58

Franks NR, Tofts C. 1994. Foraging for work-how tasks allocate workers. Anim. Behav. 48(2):470-72

Gautrais J, Theraulaz G, Deneubourg JL, Anderson C. 2002. Emergent polyethism as a consequence of increased colony size in insect societies. 7. Theor. Biol. 215(3):363-73

Gazda SK, Connor RC, Edgar RK, Cox F. 2005. A division of labour with role specialization in group-hunting bottlenose dolphins (Tursiops truncatus) off Cedar Key, Florida. Proc. R. Soc. B 272(1559):135-40

Ghirlanda S, Enquist M. 1998. Artificial neural networks as models of stimulus control. Anim. Behav. 56:138389

Gordon DM. 1991. Behavioral flexibility and the foraging ecology of seed-eating ants. Am. Nat. 138(2):379411

Gordon DM. 1996. The organization of work in social insect colonies. Nature 380(6570):121-24

Gordon DM, Goodwin BC, Trainor LEH. 1992. A parallel distributed model of the behavior of ant colonies. 7. Theor. Biol. 156(3):293-307

Gordon DM, Mehdiabadi NJ. 1999. Encounter rate and task allocation in harvester ants. Behav. Ecol. Sociobiol. 45(5):370-77

Gorelick R, Bertram SM. 2007. Quantifying division of labor: borrowing tools from sociology, sociobiology, information theory, landscape ecology, and biogeography. Insectes Soc. 54(2):105-12

Gorelick R, Bertram SM, Killeen PR, Fewell JH. 2004. Normalized mutual entropy in biology: quantifying division of labor. Am. Nat. 164(5):677-82

Gove R, Hayworth M, Chhetri M, Rueppell O. 2009. Division of labour and social insect colony performance in relation to task and mating number under two alternative response threshold models. Insectes Soc. 56(3):319-31

Graff J, Jemielity S, Parker JD, Parker KM, Keller L. 2007. Differential gene expression between adult queens and workers in the ant Lasius niger. Mol. Ecol. 16(3):675-83

Graham S, Myerscough MR, Jones JC, Oldroyd BP. 2006. Modelling the role of intracolonial genetic diversity on regulation of brood temperature in honey bee (Apis mellifera L.) colonies. Insectes Soc. 53(2):226-32

Greene MJ, Gordon DM. 2003. Social insects: Cuticular hydrocarbons inform task decisions. Nature 423(6935):32

Greene MJ, Gordon DM. 2007. Interaction rate informs harvester ant task decisions. Behav. Ecol. 18(2):451-55

Grozinger CM, Fan YL, Hoover SER, Winston ML. 2007. Genome-wide analysis reveals differences in brain gene expression patterns associated with caste and reproductive status in honey bees (Apis mellifera). Mol. Ecol. 16(22):4837-48

Halley JD, Winkler DA. 2008. Critical-like self-organization and natural selection: Two facets of a single evolutionary process? BioSystems 92(2):148-58

Hammond RL, Bruford MW, Bourke AFG. 2002. Ant workers selfishly bias sex ratios by manipulating female development. Proc. R. Soc. B 269(1487):173-78

Helms KR, Reuter M, Keller L. 2005. Sex-ratio conflict between queens and workers in eusocial Hymenoptera: mechanisms, costs, and the evolution of split colony sex ratios. Evolution 59(12):2626-38 
Hölldobler B, Wilson EO. 1990. The Ants. Berlin: Springer-Verlag

Huang ZY, Robinson GE. 1996. Regulation of honey bee division of labor by colony age demography. Bebav. Ecol. Sociobiol. 39(3):147-58

Jeanson R, Clark RM, Holbrook CT, Bertram SM, Fewell JH, Kukuk PF. 2008. Division of labour and socially induced changes in response thresholds in associations of solitary halictine bees. Anim. Behav. 76:593-602

Jeanson R, Fewell JH, Gorelick R, Bertram SM. 2007. Emergence of increased division of labor as a function of group size. Behav. Ecol. Sociobiol. 62:289-98

Jeanson R, Kukuk PF, Fewell JH. 2005. Emergence of division of labour in halictine bees: contributions of social interactions and behavioural variance. Anim. Behav. 70:1183-93

Johnson BR. 2003. Organization of work in the honeybee: a compromise between division of labour and behavioural flexibility. Proc. R. Soc. B 270(1511):147-52

Julian GE, Fewell JH. 2004. Genetic variation and task specialization in the desert leaf-cutter ant, Acromyrmex versicolor. Anim. Behav. 68:1-8

Karsai I, Wenzel JW. 1998. Productivity, individual-level and colony-level flexibility, and organization of work as consequences of colony size. Proc. Natl. Acad. Sci. USA 95(15):8665-69

Kemp AC, Woodcock M. 1995. The Hornbills: Bucerotiformes. Oxford: Oxford Univ. Press

Leoncini I, Le Conte Y, Costagliola G, Plettner E, Toth AL, et al. 2004. Regulation of behavioral maturation by a primer pheromone produced by adult worker honey bees. Proc. Natl. Acad. Sci. USA 101(50):17559-64

MacKay WP. 1982. The effect of predation of western widow spiders (Araneae, Theridiidae) on harvester ants (Hymenoptera, Formicidae). Oecologia 53(3):406-11

Mailleux AC, Deneubourg JL, Detrain C. 2003. How does colony growth influence communication in ants? Insectes Soc. 50(1):24-31

Manser MB. 1999. Response of foraging group members to sentinel calls in suricates, Suricata suricatta. Proc. R. Soc. B 266(1423):1013-19

Maynard Smith J. 1982. Evolution and the Theory of Games. Cambridge, UK: Cambridge Univ. Press

McNamara JM, Weissing FJ. 2010. Evolutionary game theory. In Social Behaviour: Genes, Ecology and Evolution, ed. T Székely, AJ Moore, J Komdeur, pp. 109-33. Cambridge, UK: Cambridge Univ. Press

Mertl AL, Traniello JFA. 2009. Behavioral evolution in the major worker subcaste of twig-nesting Pheidole (Hymenoptera: Formicidae): Does morphological specialization influence task plasticity? Behav. Ecol. Sociobiol. 63(10):1411-26

Mitri S, Floreano D, Keller L. 2009. The evolution of information suppression in communicating robots with conflicting interests. Proc. Natl. Acad. Sci. USA 106(37):15786-90

Mitri S, Floreano D, Keller L. 2011. Relatedness influences signal reliability in evolving robots. Proc. R. Soc. B 278(1704):378-83

Munger JC. 1984. Long-term yield from harvester ant colonies-implications for horned lizard foraging strategy. Ecology 65(4):1077-86

Muscedere ML, Willey TA, Traniello JFA. 2009. Age and task efficiency in the ant Pheidole dentata: young minor workers are not specialist nurses. Anim. Behav. 77(4):911-18

Naug D, Gadagkar R. 1999. Flexible division of labor mediated by social interactions in an insect colony-a simulation model. 7. Theor. Biol. 197(1):123-33

Nee S. 1987. The evolution of multicompartmental genomes in viruses. F. Mol. Evol. 25(4):277-81

Nowak M. 1990. An evolutionarily stable strategy may be inaccessible. F. Theor. Biol. 142(2):237-41

O'Donnell S, Foster RL. 2001. Thresholds of response in nest thermoregulation by worker bumble bees, Bombus bifarius nearcticus (Hymenoptera: Apidae). Ethology 107(5):387-99

Oster GF, Wilson EO. 1978. Caste and Ecology in the Social Insects. Princeton, NJ: Princeton Univ. Press

Pacala SW, Gordon DM, Godfray HCJ. 1996. Effects of social group size on information transfer and task allocation. Evol. Ecol. 10:127-65

Page RE, Amdam GV. 2007. The making of a social insect: developmental architectures of social design. BioEssays 29(4):334-43

Page RE, Erber J, Fondrk MK. 1998. The effect of genotype on response thresholds to sucrose and foraging behavior of honey bees (Apis mellifera L.). F. Comp. Physiol. A 182(4):489-500

Page RE, Mitchell SD. 1998. Self-organization and the evolution of division of labor. Apidologie 29(1-2):171-90 
Pankiw T, Page RE, Fondrk MK. 1998. Brood pheromone stimulates pollen foraging in honey bees (Apis mellifera). Behav. Ecol. Sociobiol. 44(3):193-98

Passera L, Aron S, Vargo EL, Keller L. 2001. Queen control of sex ratio in fire ants. Science 293(5533):1308-10

Pen I, Taylor PD. 2005. Modelling information exchange in worker-queen conflict over sex allocation. Proc. R. Soc. B 272(1579):2403-8

Perez-Uribe A, Floreano D, Keller L. 2003. Effects of group composition and level of selection in the evolution of cooperation in artificial ants. Lect. Notes Comput. Sci. 2801:128-37

Plowright RC, Plowright CMS. 1988. Elitism in social insects: a positive feedback model. In Interindividual Behavioral Variability in Social Insects, ed. RL Jeanne, pp. 419-31. Boulder, CO: Westview

Pratt SC. 2005. Quorum sensing by encounter rates in the ant Temnothorax albipennis. Behav. Ecol. 16(2):488-96

Ratnieks FLW. 2001. Heirs and spares: caste conflict and excess queen production in Melipona bees. Behav. Ecol. Sociobiol. 50(5):467-73

Ratnieks FLW, Foster KR, Wenseleers T. 2006. Conflict resolution in insect societies. Annu. Rev. Entomol. 51:581-608

Ravary F, Lecoutey E, Kaminski G, Chaline N, Jaisson P. 2007. Individual experience alone can generate lasting division of labor in ants. Curr. Biol. 17(15):1308-12

Reuter M, Helms KR, Lehmann L, Keller L. 2004. Effects of brood manipulation costs on optimal sex allocation in social Hymenoptera. Am. Nat. 164(3):E73-82

Reuter M, Keller L. 2001. Sex ratio conflict and worker production in eusocial Hymenoptera. Am. Nat. 158(2):166-77

Richardson RC. 2001. Complexity, self-organization and selection. Biol. Philos. 16(5):655-83

Robinson EJH, Feinerman O, Franks NR. 2009a. Flexible task allocation and the organization of work in ants. Proc. R. Soc. B 276(1677):4373-80

Robinson EJH, Richardson TO, Sendova-Franks AB, Feinerman O, Franks NR. 2009b. Radio tagging reveals the roles of corpulence, experience and social information in ant decision making. Behav. Ecol. Sociobiol. 63(5):627-36

Robinson GE. 1987. Regulation of honey bee age polyethism by juvenile hormone. Behav. Ecol. Sociobiol. 20(5):329-38

Robinson GE. 1992. Regulation of division of labor in insect societies. Annu. Rev. Entomol. 37:637-65

Robinson GE, Page RE. 1989. Genetic determination of nectar foraging, pollen foraging, and nest-site scouting in honey bee colonies. Behav. Ecol. Sociobiol. 24(5):317-23

Robinson GE, Page RE, Huang ZY. 1994. Temporal polyethism in social insects is a developmental process. Anim. Behav. 48(2):467-69

Rosset H, Keller L, Chapuisat M. 2005. Experimental manipulation of colony genetic diversity had no effect on short-term task efficiency in the Argentine ant Linepithema humile. Behav. Ecol. Sociobiol. 58(1):87-98

Schafer RJ, Holmes S, Gordon DM. 2006. Forager activation and food availability in harvester ants. Anim. Behav. 71:815-22

Schmid-Hempel P. 1992. Worker castes and adaptive demography. F. Evol. Biol. 5(1):1-12

Schulz DJ, Huang ZY, Robinson GE. 1998. Effects of colony food shortage on behavioral development in honey bees. Behav. Ecol. Sociobiol. 42(5):295-303

Seeley TD. 2002. When is self-organization used in biological systems? Biol. Bull. 202(3):314-18

Seeley TD, Morse RA. 1978. Nest site selection by the honey bee, Apis mellifera. Insectes Soc. 25(4):323-37

Seeley TD, Tovey CA. 1994. Why search time to find a food-storer bee accurately indicates the relative rates of nectar collecting and nectar processing in honey bee colonies. Anim. Behav. 47(2):311-16

Seid MA, Traniello JFA. 2006. Age-related repertoire expansion and division of labor in Pheidole dentata (Hymenoptera: Formicidae): a new perspective on temporal polyethism and behavioral plasticity in ants. Behav. Ecol. Sociobiol. 60(5):631-44

Smith A. 1776. An Inquiry into the Nature and Causes of the Wealth of Nations. London: W. Strahan and T. Cadell

Smith CD, Smith CR, Mueller U, Gadaus J. 2010. Ant genomics: strength and diversity in numbers. Mol. Ecol. 19(1):31-35

Snyder LE. 1992. The genetics of social behavior in a polygynous ant. Naturwissenschaften 79(11):525-27 
Stuart RJ, Page RE. 1991. Genetic component to division of labor among workers of a leptothoracine ant. Naturwissenschaften 78(8):375-77

Sundström L, Chapuisat M, Keller L. 1996. Conditional manipulation of sex ratios by ant workers: a test of kin selection theory. Science 274(5289):993-95

Szathmáry E, Maynard Smith J. 1995. The major evolutionary transitions. Nature 374(6519):227-32

Tannenbaum E. 2007. When does division of labor lead to increased system output? 7. Theor. Biol. 247(3):41325

Tarapore D, Floreano D, Keller L. 2009. Task dependent influence of genetic architecture and mating frequency on division of labour in social insect societies. Behav. Ecol. Sociobiol. 64(4):675-84

Tarpy DR. 2003. Genetic diversity within honeybee colonies prevents severe infections and promotes colony growth. Proc. R. Soc. B 270(1510):99-103

Theraulaz G, Bonabeau E, Deneubourg JL. 1998. Response threshold reinforcement and division of labour in insect societies. Proc. R. Soc. B 265(1393):327-32

Thomas ML, Elgar MA. 2003. Colony size affects division of labour in the ponerine ant Rhytidoponera metallica. Naturwissenschaften 90(2):88-92

Tofts C. 1993. Algorithms for task allocation in ants. (A study of temporal polyethism: theory). Bull. Math. Biol. 55(5):891-918

Traniello JFA, Rosengaus RB. 1997. Ecology, evolution and division of labour in social insects. Anim. Behav. 53:209-13

Visscher PK. 2007. Group decision making in nest-site selection among social insects. Annu. Rev. Entomol. 52:255-75

Waddington SJ, Santorelli LA, Ryan FR, Hughes WOH. 2010. Genetic polyethism in leaf-cutting ants. Behav. Ecol. 21(6):1165-69

Wahl LM. 2002. Evolving the division of labour: generalists, specialists and task allocation. F. Theor. Biol. 219(3):371-88

Waibel M, Floreano D, Magnenat S, Keller L. 2006. Division of labour and colony efficiency in social insects: effects of interactions between genetic architecture, colony kin structure and rate of perturbations. Proc. R. Soc. B 273:1815-23

Wakano JYK, Nakata K, Yamamura N. 1998. Dynamic model of optimal age polyethism in social insects under stable and fluctuating environments. F. Theor. Biol. 193:153-65

Weidenmuller A. 2004. The control of nest climate in bumblebee (Bombus terrestris) colonies: interindividual variability and self reinforcement in fanning response. Behav. Ecol. 15(1):120-28

Weidenmuller A, Mayr C, Kleineidam CJ, Roces F. 2009. Preimaginal and adult experience modulates the thermal response behavior of ants. Curr. Biol. 19(22):1897-902

Wenseleers T, Ratnieks FLW, Billen J. 2003. Caste fate conflict in swarm-founding social Hymenoptera: an inclusive fitness analysis. 7. Evol. Biol. 16(4):647-58

Wilson EO. 1971. The Insect Societies. Cambridge, MA: Harvard Univ. Press

Wilson EO. 1980. Caste and division of labor in leaf-cutter ants (Hymenoptera, Formicidae, Atta). 1. The overall pattern in Atta sexdens. Behav. Ecol. Sociobiol. 7(2):143-56

Wilson EO. 1985. Between-caste aversion as a basis for division of labor in the ant Pheidole pubiventris (Hymenoptera, Formicidae). Behav. Ecol. Sociobiol. 17(1):35-37

Wilson-Rich N, Spivak M, Fefferman NH, Starks PT. 2009. Genetic, individual, and group facilitation of disease resistance in insect societies. Annu. Rev. Entomol. 54:405-23

Wurm Y, Wang J, Keller L. 2010. Changes in reproductive roles are associated with changes in gene expression in fire ant queens. Mol. Ecol. 19(6):1200-11

Yang AS. 2006. Seasonality, division of labor, and dynamics of colony-level nutrient storage in the ant Pheidole morrisi. Insectes Soc. 53(4):456-62 
Annual Review of Ecology, Evolution, and Systematics

\section{Contents}

Volume 42, 2011

Native Pollinators in Anthropogenic Habitats

Rachael Winfree, Ignasi Bartomeus, and Daniel P. Cariveau 1

Microbially Mediated Plant Functional Traits

Maren L. Friesen, Stephanie S. Porter, Scott C. Stark, Eric 7. von Wettberg, foel L. Sachs, and Esperanza Martinez-Romero

Evolution in the Genus Homo

Bernard Wood and Fennifer Baker

Ehrlich and Raven Revisited: Mechanisms Underlying Codiversification of Plants and Enemies

Niklas fanz

An Evolutionary Perspective on Self-Organized Division of Labor in Social Insects Ana Duarte, Franz 7. Weissing, Ido Pen, and Laurent Keller

Evolution of Anopheles gambiae in Relation to Humans and Malaria Bradley 7. White, Frank H. Collins, and Nora 7. Besansky

Mechanisms of Plant Invasions of North America and European Grasslands T.R. Seastedt and Petr Pyšek

Physiological Correlates of Geographic Range in Animals

Francisco Bozinovic, Piero Calosi, and Fohn I. Spicer

Ecological Lessons from Free-Air $\mathrm{CO}_{2}$ Enrichment (FACE) Experiments

Richard 7. Norby and Donald R. Zak

Biogeography of the Indo-Australian Archipelago

David 7. Lohman, Mark de Bruyn, Timothy Page, Kristina von Rintelen,

Robert Hall, Peter K.L. Ng, Hsi-Te Shih, Gary R. Carvalho, and Thomas von Rintelen

Phylogenetic Insights on Evolutionary Novelties in Lizards and Snakes: Sex, Birth, Bodies, Niches, and Venom Fack W. Sites Fr, Tod W. Reeder, and Fobn 7. Wiens 
The Patterns and Causes of Variation in Plant Nucleotide Substitution Rates

Brandon Gaut, Liang Yang, Shobei Takuno, and Luis E. Eguiarte

Long-Term Ecological Records and Their Relevance to Climate Change

Predictions for a Warmer World

K.7. Willis and G.M. MacDonald

The Behavioral Ecology of Nutrient Foraging by Plants

James F. Cabill 7 r and Gordon G. McNickle

Climate Relicts: Past, Present, Future

Arndt Hampe and Alistair S. Fump

Rapid Evolutionary Change and the Coexistence of Species

Richard A. Lankau

Developmental Patterns in Mesozoic Evolution of Mammal Ears

Zhe-Xi Luo

Integrated Land-Sea Conservation Planning: The Missing Links

Forge G. Álvarez-Romero, Robert L. Pressey, Natalie C. Ban, Ken Vance-Borland,

Chuck Willer, Carissa Foy Klein, and Steven D. Gaines

On the Use of Stable Isotopes in Trophic Ecology

William 7. Boecklen, Christopher T. Yarnes, Bethany A. Cook, and Avis C. Fames

Phylogenetic Methods in Biogeography

Fredrik Ronquist and Isabel Sanmartin

Toward an Era of Restoration in Ecology: Successes, Failures, and Opportunities Ahead

Katharine N. Suding

Functional Ecology of Free-Living Nitrogen Fixation:

A Contemporary Perspective

Sasha C. Reed, Cory C. Cleveland, and Alan R. Townsend

\section{Indexes}

Cumulative Index of Contributing Authors, Volumes 38-42

Cumulative Index of Chapter Titles, Volumes 38-42

\section{Errata}

An online log of corrections to Annual Review of Ecology, Evolution, and Systematics articles may be found at http://ecolsys.annualreviews.org/errata.shtml 